\title{
Pole Tilt Monitoring System Using MEMs Accelerometer and Beaglebone Black
}

\author{
Lok De Yong, Nurul Asyikin Mohamed Radzi, Yanti Erana Jalil
}

Universiti Tenaga Nasional, Jalan Ikram-Uniten, 43000 Kajang, Selangor, Malaysia

\begin{tabular}{l}
\hline \hline Article Info \\
\hline Article history: \\
Received Feb 1, 2018 \\
Revised Apr 21, 2018 \\
Accepted Apr 27, 2018 \\
\hline
\end{tabular}

\section{Keywords:}

BeagleBone Black MEMs accelerometer Tilt angle.

\begin{abstract}
Electric pole could tilt in the event of heavy storms, soil erosion and object crashing onto poles, causing serious damages if they are not fixed before condition worsen. Therefore, to monitor the health of the electric pole, a system to monitor its tilting angle is needed. In this paper, for the first time, to the best of our knowledge, an integration of Micro Electro-Mechanical Systems (MEMs) accelerometer with BeagleBone Black microcomputer will be used to develop an electric pole tile angle monitoring system. The accuracy of measuring tilt angle using single axis accelerometer versus triple axis accelerometer and the effect of rolling on tilt angle reading using single axis accelerometer versus triple axis accelerometer will be presented. Threeaxis accelerometer achieves greater accuracy as the percentage error of tilting and rolling for the three-axis accelerometer are better than the single-axis accelerometer.
\end{abstract}

Copyright (C) 2018 Institute of Advanced Engineering and Science. All rights reserved.

\section{Corresponding Author:}

Nurul Asyikin Mohamed Radzi, Universiti Tenaga Nasional, Jalan Ikram-Uniten, 43000 Kajang, Selangor, Malaysia.

Email: asyikin@uniten.edu.my

\section{INTRODUCTION}

Electric pole could tilt in the event of heavy storms, soil erosion, object crashing onto poles and other unforeseen circumstances. Tilted electric poles are dangerous to living beings and could cause serious damages if they are not fixed before condition worsen. Furthermore, any electric pole that tilts more than its allowable angle could collapse to the ground easily.

Therefore, to monitor the health of the electric pole, a system to monitor its tilting angle is needed. One of the most important components in monitoring the tilting angle is sensor. To date, most researchers use accelerometer as the sensor to study the tilt angle [1-7]. Luczak et al. [1] Stated that a tilting angle is able to be measured by using components of gravitational acceleration. The accelerometer must contain at least three single axis in order to measure all possible position with respect to gravity vector. By adjusting the tilt sensor, a proper gravitational accelerator is able to be determined. The paper agrees that Micro Electro-Mechanical Systems (MEMs) accelerometer is suitable to be used as tilt sensor and has proven that gravitation vector is able to be used to find the tilting angle.

Qian et al. worked on sensing tilt accuracy with a tilt sensing scheme, Linear Model proposed by using accelerometers [2]. In this paper, the gravitational acceleration of the earth is broken down into three components. Three numerical models were proposed to sense the tilting angle accurately.

MEMs accelerometer have also been used to study numerous other applications. Takao et al. utilized the ccelerometer for earthquake sensing device in Japan [3]. On the other hand, H. Gjoreski and M. Gams used accelerometer to recognize posture and to detect fall [4]. Four sensors were placed on different parts of the body to recognize the posture of a human. The experiment was conducted with situation that falls and non-falls were hard to be recognized. It is proven that three accelerometers were able to recognize all types of 
falling expect slow fall. Whereas, two accelerometers were able to achieve $90 \%$ accuracy in posture recognition and one accelerometer was only able to differentiate clear-cut fall.

With the proven advantages of MEMs accelerometer in measuring tilt angle in various applications, this paper will investigate its suitability in measuring the tilt angle for electric pole. This is because; there is yet a study on applying MEMs accelerometer to measure the tilt angle.

Another important component in developing the system to monitor electric pole, is microcomputer. Microcomputer is one of the most cost efficient methods to be used considering the large number of electric poles. Numerous microcomputers exist in the market, among them are BeagleBone Black (BBB), Raspberry $\mathrm{Pi}$ and Arduino [8]. Although the first generation of BBB and Raspberry Pi are released in the same year, researches and publications on BBB are still very limited. To date, while 40,500 results are found in Google Scholar on Raspberry Pi, only 3,310 are found on BBB.

Some of the studies done using BBB is reviewed as follows. In [9], Yocto project is proposed to build a free custom Operating System (OS) which will support Internet of Things in BBB as low cost computing platform. In [10], Sagar et al. integrates tilt sensor and accelerometer with BBB for automotive monitoring system to analyze car condition during a crash incident. In [11], an underwater acoustical data is recorded using BBB. In [12], electronic sensors and actuator technologies are also mounted on harness for cyber enhanced working dog that is used to handle sensor information and communication from handlers.

Besides that, ABES Engineering College used BBB to run system prototype which bridge the gap in communication process between the blind, deaf and dumb people [13]. Researchers from University of Freiburg, Germany used BBB integrated with expansion circuit board and compatible touchscreen as indoor handheld device for emergency responders [14]. Researchers from University of Naples Federico II, Italy applies BBB in designing smart museum project. Two types of sensor nodes which are server node and slave node are integrated with BBB for the realization of Internet of Things (IoT) [15].

Unlike Raspberry Pi and Arduino, the BBB contains analog read pin which measures voltage level from 0 to $1.8 \mathrm{~V}$. The availability of analog read pin on the BBB makes the integration of MEMs accelerometer sensors with BBB possible. Therefore, in this paper, for the first time, to the best of our knowledge, an integration of MEMs accelerometer with BBB microcomputer will be used to develop an electric pole tile angle monitoring system. The accuracy of measuring tilt angle using single axis accelerometer versus triple axis accelerometer and the effect of rolling on tilt angle reading using single axis accelerometer versus triple axis accelerometer will also be done.

The rest of the paper will be divided as follows. Section 2 will be discussing on the electric pole monitoring system, Section 3 on performance measurement and analysis and Section 4 on conclusion.

\section{POLE TILT MONITORING SYSTEM MODEL}

The electric pole tilt monitoring system proposed consist of two important components which are ADXL 335 MEMs accelerometer as sensor and BBB as microcomputer. Other components used are SIM900a Global System for Mobile Communications (GSM) module as communication device, Universal Serial Bus (USB) to universal asynchronous receiver-transmitter (UART) converter powered by FTDI FT232RL integrated circuit (IC), and UART to RS232 converter powered by MAX232 IC for remote monitoring.

An accelerometer is an electronic device used to sense acceleration. The accelerometer is basically built with a seismic mass attached to the housing. The gravitational acceleration causes the spring attached to the seismic mass to stretch downwards.

Tilt angle can be measured by using components of gravitational acceleration. The accelerometer must at least contain three single axis in order to measure all possible position with respect to gravity vector. By adjusting the tilt sensor, a proper gravitational accelerator can be determined [1].

BBB is a microcomputer with the size of a debit card introduced in 2013. Despite its small size, it is powerful and able to run full desktop applications. The BBB is preloaded with Linux OS that serves up a web-based integrated development environment (IDE) known as Cloud9. The board functions as a programmable cloud all on its own.

There are mainly two expansion headers called P8 and P9 on the BBB. These two headers provide extensive input/output (I/O) capabilities to the board. These expansion headers contains 65 possible digital I/Os, eight Pulse Width Modulations, four timers, seven analog inputs (1.8V), four UARTs, and other pins. The P9 headers also have 5V and 3.3V voltage supplies. Both P8 and P9 headers have GND. 
Figure 1 shows the pole tilt monitoring system model used in this paper, with block diagram shown in Figure 2. From this model, the ADXL335 converts gravitational force to voltage readings. The voltage readings from the ADXL335 are then passed to analog read pins of the BBB. Since the analog read pins of the board is only $1.8 \mathrm{~V}$ tolerance, potentiometers are used to reduce the output voltage of the ADXL335 MEMs accelerometer to prevent damage to the analog read pins. The pole tilt monitoring system parameters is shown in Table 1.

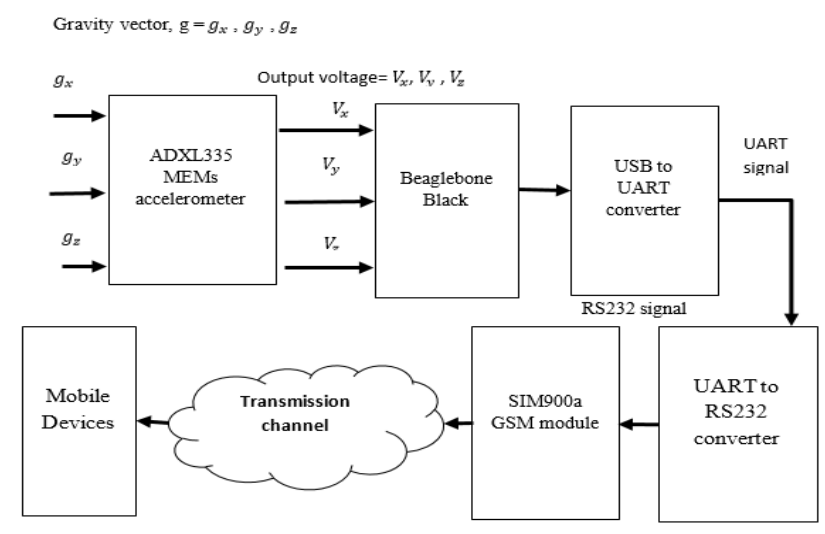

Figure 1. Pole Tilt Monitoring System Model
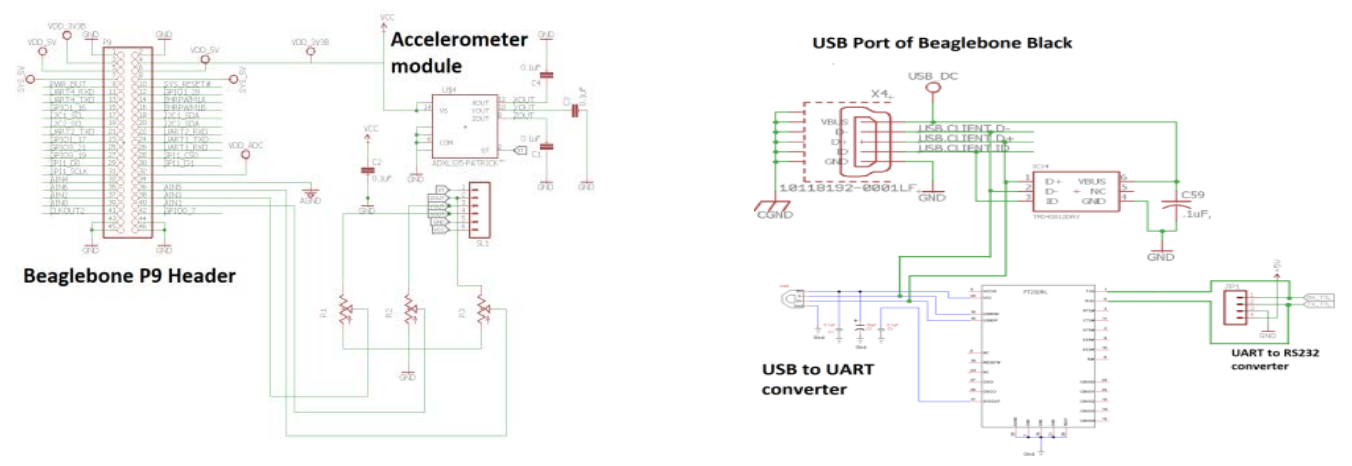

Figure 2. Physical Wired Connection of the Prototype

Table 1. Pole Tilt Monitoring System Parameters

\begin{tabular}{lll}
\hline Parameter & Minimum & Maximum \\
\hline Raw analog read pin value & 0.66 & 0.99 \\
Normalized X,Y,Z value & -1 & 1 \\
Pole tilt angle, $\theta$ & $0^{\circ}$ & $90^{\circ}$ \\
Pin read interval (seconds) & $2 \mathrm{~s}$ & \\
\hline
\end{tabular}

The BBB is installed with Python IDE. Therefore, Python programming language is used to control the analog read pins and USB ports of the board. The Python code is also used to perform mathematical calculation to derive the tilt angle using reading from the analog read pins. Serial port library in the Python IDE is used to send AT Commands to the SIM900a GSM module when electric poles tilts more than its allowable preset tilt angle. The instructions of sending short messaging system (SMS) will be sent via the USB ports of BBB. The USB signal will be further converted to UART signal with USB to UART converter. Then, the UART signal will be converted to RS232 signal using UART to RS232 converter before it can be passed to the SIM900a GSM module. A subscriber identification module (SIM) card will need to be installed on the SIM900a GSM module to access service provided by Telco companies. With SIM Card installed onto the SIM900a GSM module, the SIM900a will send an alert SMS to the registered phone. 
By finding the minimum and maximum limit of the value registered by the analog read pin, linear equation (1) is derived using simultaneous linear equation. Linear equation is used to convert to raw analog read pin value to normalized $\mathrm{X}, \mathrm{Y}, \mathrm{Z}$ value.

$$
y(t)=6.06 x(t)-5
$$

where $y(t)$ is the normalized value of $\mathrm{X}, \mathrm{Y}, \mathrm{Z}$ and $x(t)$ is the raw analog read pin value.

To obtain the resultant value of X, Y, Z, Equation (2) is used [16]

$$
r(t)=\sqrt{y_{x}(t)^{2}+y_{y}(t)^{2}+y_{z}(t)^{2}}
$$

where $r(\mathrm{t})=$ resultant value of $\mathrm{X}, \mathrm{Y}, \mathrm{Z}$ and $y(t)=$ normalized value of $X, Y, Z$. The $\mathrm{r}(\mathrm{t})$ value will be used to calculate the cosine value, by taking the normalized value of $Z, y_{Z}(t)$ divided by the resultant value of $X$, Y, $\mathrm{Z}, r(t)$. Hence, the cosine function will be [3]:

$$
\cos (\theta)=\frac{y_{z}(t)}{r(t)}
$$

To obtain the tilted angle of an electric pole, the cosine function in Equation (3) is inversed to become:

$$
\theta=\cos ^{-1}\left(\frac{y_{z}(t)}{r(t)}\right)
$$

where $\theta$ is the tilted angle of an electric pole. By default, the electric pole is always standing upright, $90^{\circ}$, therefore the tilt angle of an electric pole will be:

$$
\text { tilt angle }=90^{\circ}-\theta
$$

\section{PERFORMANCE MEASUREMENT AND ANALYSIS}

In this section, the accuracy of measuring tilt angle using single axis accelerometer versus triple axis accelerometer are being investigated. The effect of rolling on tilt angle reading using single axis accelerometer versus triple axis accelerometer will also be presented. 16 tilt angles are measured using single axis and three-axis accelerometer.

The measured tilt angles for single axis and three-axis accelerometer are tabulated in Table 2 and 3 respectively. The percentage error is then calculated by:

$$
\% \text { error }=\frac{\mid \text { measured angle-actual angle } \mid}{90} \times 100 \%
$$

The measured tilt angles for both single axis and three-axis accelerometer are then compared to the actual tilt angle by plotting a graph of measured tilt angle versus actual tilt angle as shown in Figure 3. It can be seen that the graph of three-axis accelerometer is closer to the ideal graph compared to that of one-axis accelerometer. When the tilt angle is below $70^{\circ}$, both accelerometers show about the same performance. However, once the accelerometer exceeds $70^{\circ}$, three-axis accelerometer starts to show more accurate readings than the one-axis accelerometer. 
Table 2. Measured Tilt Angle and Percentage Error of Single Axis Accelerometer

\begin{tabular}{ccc}
\hline Actual tilt angle, $^{\circ}$ & Measured tilt angle, ${ }^{\circ}$ & Percentage error, $\%$ \\
\hline 0 & 0.311 & 0.3456 \\
5 & 3.98 & 1.13 \\
10 & 10.99 & 1.1 \\
15 & 15.75 & 0.83 \\
20 & 21.8 & 2.00 \\
25 & 26.5 & 1.67 \\
35 & 36.51 & 1.68 \\
40 & 39.7 & 0.33 \\
50 & 50.9 & 1.00 \\
55 & 55.15 & 0.167 \\
65 & 64.8 & 0.22 \\
70 & 69 & 1.11 \\
75 & 71 & 4.44 \\
80 & 74 & 6.67 \\
85 & 76 & 10.00 \\
90 & 79 & 12.22 \\
\hline
\end{tabular}

Table 3. Measured Tilt Angle and Percentage Error of three Axis Accelerometer

\begin{tabular}{ccc}
\hline Actual tilt angle, $^{\circ}$ & Measured tilt angle, $^{\circ}$ & Percentage error, $\%$ \\
\hline 0 & 0.32 & 0.35 \\
5 & 5.97 & 1.08 \\
10 & 11.13 & 1.26 \\
15 & 15.83 & 0.92 \\
20 & 22.5 & 2.78 \\
25 & 27.5 & 2.78 \\
35 & 37.9 & 3.22 \\
40 & 43.15 & 3.50 \\
50 & 53.38 & 3.76 \\
65 & 67.11 & 2.34 \\
70 & 73.8 & 4.22 \\
75 & 78.9 & 4.33 \\
80 & 82 & 2.22 \\
85 & 86.13 & 1.26 \\
90 & 87.84 & 2.40 \\
\hline
\end{tabular}

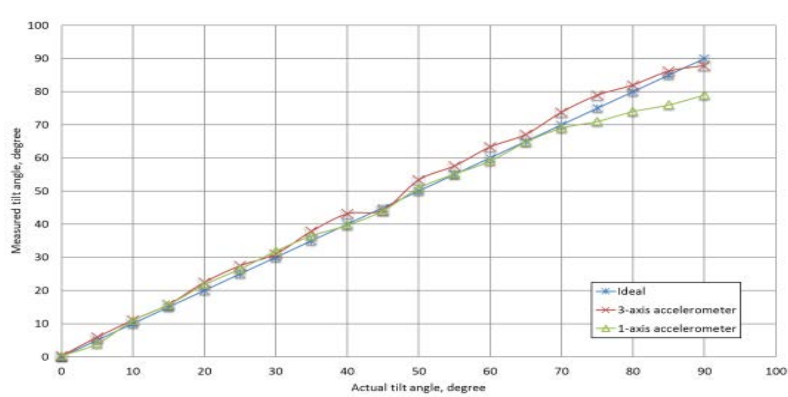

Figure 3. Comparison Between the Accuracy of Single Axis Accelerometer and three-Axis Accelerometer

The single axis accelerometer registers maximum percentage error of $12.22 \%$ at $90^{\circ}$. The three axis accelerometer registers maximum percentage error of $4.33 \%$ at $75^{\circ}$. The three-axis accelerometer is also more reliable compared to single-axis accelerometer. This is because the three axis accelerometer registers more consistent reading compared to single axis accelerometer. The inconsistent reading registered by the single axis accelerometer can cause mathematical error when deriving tilt angle. Unlike single axis accelerometer, the three axis accelerometer has more sensitive axis to maintain the consistency of the reading.

In short, the three-axis accelerometer achieves greater accuracy as the percentage error of tilting and rolling for the three-axis accelerometer are better than the single-axis accelerometer. The results obtained from this experiment also comes into agreement with the statement made by [2] that MEMS accelerometer can be used as tilt sensor with accuracy of approximately tenth degree. In [2], experiments were conducted and managed to prove that the theory of using gravitation vector to find the tilting angle is possible. 
The experiments in [2] were conducted with physical model of tilt sensor and using other metrological parameters.

The factors which cause the percentage error in the accelerometer reading are mainly due to the sensitive axis of accelerometer cannot be mounted perfectly aligned with the central axis of the pole [4]. Besides that, it is believed that there is a little bending on the pole which could contribute to the increase of percentage error. The percentage error is also believed to be caused by non-liner relation between the gravitational acceleration and the tilt angle [4].

Other than those major factors mentioned above, there are also some minor factors which could cause very little percentage error in the accelerometer reading. The first minor factor is believed to be the noise in the analog read pin of the BBB which could cause less than $1 \%$ fluctuation in the voltage reading. The second minor factor is the rounding off decimals when deriving the linear equation algorithm for normalization. Thirdly, the inconsistent adjustment of rheostat connected to the accelerometer could also cause slight deviation of measured readings. Then, the longer jumper wire connections could also cause voltage drop which are believed to contribute to the minor increase in percentage error. Despite all these minor factors mentioned, they do not bring significant impact to the accuracy in the result obtained thus they can be neglected.

For the analysis on the effect of rolling on accelerometer, the accelerometer is placed horizontally on a table. The accelerometer attached to the pole was rolled slowly. The changes in tilt angle measured by the single axis and three-axis accelerometer were observed and recorded as shown in Figure 4 and Figure 5 respectively. The minimum and maximum value of the measured tilt angle was selected to determine the effects of rolling on the accelerometer. The Python code was programmed to register the accelerometer reading every second so that more data can be recorded to investigate the effect of rolling.

Based on Figure 4, it can be seen that the measured angle increase from approximately -2.397 degree to 4.271 degree as the accelerometer is slowly rolled with the pole. The measured angle returns to approximately -2.397 degree when the accelerometer completes one revolution. From Figure 5, it is identified that the maximum measured tilt angle is 4.2713 degree. While the minimum measured tilt angle is -3.3067 degree. Therefore, the range of tilt angle due to the effect of rolling can be expressed as:

$$
-3.3067^{\circ} \leq \text { changes of tilt angle due to rolling } \leq 4.2713^{\circ}
$$

In short, the effect of rolling on single axis accelerometer can cause up to $8.4201 \%$ deviation from the actual tilt angle of 0 degree.

Based on Figure 5, it can be seen that the measured angle decrease from approximately 1.68 degree to 1.108 degree as the accelerometer is slowly rolled with the pole. The tilt angle then increase from 1.108 degree all the way to 5.75 degree. The measured angle returns to approximately 1.6734 degree when the accelerometer completes one revolution. From Figure 5, it is identified that the maximum measured tilt angle is 5.7594 degree. While the minimum measured tilt angle is 1.1088 degree. Therefore, the range of tilt angle due to the effect of rolling can be expressed as:

\section{$1.1088^{\circ} \leq$ changes of tilt angle due to rolling $\leq 5.7594^{\circ}$}

The effect of rolling on three axis accelerometer can cause up to 5.1673\% deviation from the actual tilt angle of 0 degree. The delay of the MEMs accelerometer is defined as the time taken for accelerometer sensor to register stable reading after tilt. It is found out that the delay is approximately 1.3 seconds.

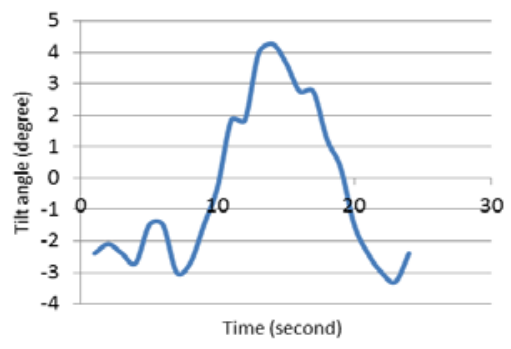

Figure 4. Changes of Tilt Angle when Single Axis Accelerometer was Rolled Along with the Pole

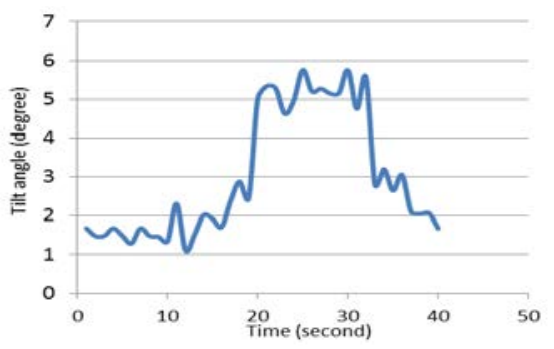

Figure 5. Changes of Tilt Angle when three Axis Accelerometer was Rolled Along with the Pole 


\section{SUMMARY AND CONCLUSION}

In conclusion, the integration of ADXL335 MEMs accelerometer with BBB is suitable to be used as electric pole tilt monitoring system. The accuracy of measuring tilt angle using single axis accelerometer versus triple axis accelerometer are being investigated. It shows that to achieve greater accuracy, three-axis accelerometer should be used instead of single axis accelerometer. The effect of rolling on tilt angle reading using single axis accelerometer versus triple axis accelerometer has also been presented. When the pole tilts within the preset angle, a SMS alert will be sent via SIM900a GSM module as an early warning system to warn the electric pole maintenance team.

\section{REFERENCES}

[1] Luczak, S., Oleksiuk, W., Bodnicki, M. Sensing tilt with MEMS accelerometers. IEEE Sensors Journal. 2006; 6(6): 1669-1675.

[2] Qian, J., Bin F., Weibin Y., Xiao L., Hai N. Accurate tilt sensing with linear model. IEEE Sensors Journal. 2011; 11(10): 2301-2309.

[3] Takao, A., Toshinori, K., Toshifumi, M., Tetsuya, T., Youichi, A. Application of MEMS accelerometer to geophysics. International Journal of the JCRM. 2009; 4(2): 33-36.

[4] Gjoreski, H., Mitja, L., Matjaz, G. Accelerometer placement for posture recognition and fall detection. 7th International Conference on Intelligent environments (IE). 2011; 47-54.

[5] Wang, Y., Zhang, H., Zhou, Q. Adaptive integrated navigation filtering based on accelerometer calibration. Indonesian Journal of Electrical Engineering and Computer Science. 2012; 10(7): 1869-1878.

[6] Chang, D. T. T., Tsai, Y. S., Yang, K. C. Study of Real-Time Slope Stability Monitoring System Using Wireless Sensor Network (WSN). Indonesian Journal of Electrical Engineering and Computer Science. 2013; 11(3): 14781488.

[7] Shiju, D. Wilfred. Design of Android Based Smart Car. Indonesian Journal of Electrical Engineering and Computer Science. 2017; 8(3): 648-650.

[8] Radzi, N.A.M., Ismail, A., Karunanithi, S., Weng, L.Y., Jern, K.P., Hock, G.C., Jamaluddin, J.E., Krishnan, P.S. Integrating programming with BeagleBone Black for undergraduate's programming for engineers syllabus. IEEE 8th International Conference on Engineering Education (ICEED). 2016; 12-15.

[9] Subravelu, T., Ruhan Bevi, A. Designing a custom OS for IoT using Yocto project and creating IoT apps using IBM Bluemix. International Journal of Advances in Engineering Research (IJAER). 2015; 9(6).

[10] Sagar, T.S.J., Balamurugan, M.S. Vivek, J.A. A wireless framework for automotive monitoring systems. Indian Journal of Science and Technology. 2015; 8(19).

[11] Ben, T., Andrew M., David M. Using low cost single-board microcontrollers to record underwater acoustical data. Inter-noise. 2014; 1-8.

[12] Bozkurt, A., Roberts, D., Sherman, B., Brugarolas, R., Mealin, S., Majikes, J., Yang, P., Loftin, R. Toward CyberEnhanced Working Dogs for Search and Rescue. IEEE Intelligent Systems. 2014; 29(6): 32-39.

[13] Rastogi, R., Mittal, S., Agarwal, S. A Novel Approach for Communication among Blind, Deaf and Dumb People. 2nd International Conference on Computing for Sustainable Global Development. 2013; 2227-2232.

[14] Simon, N., Bordoy, J., Höflinger, F., Wendeberg, J., Schink, M., Tannhäuser, R., Reindl L., Schindelhauer. C. Indoor Localization System for Emergency Responders with Ultra Low-power Radio Landmarks. IEEE Instrumentation and Measurement Technology Conference. 2015; 1-6.

[15] Chianese, A. and Piccialli, F. Designing a smart museum: when Cultural Heritage joins IoT. Eighth International Conference on Next Generation Mobile Apps, Services and Technologies. Oxford, UK, 2014; 1-7.

[16] M. Pedley, Tilt sensing using a Three-Axis Accelerometer. 6th ed. Denver, Colorado 80217: Freescale Semiconductor, Inc. 2013; 1-7, 9, 18. 